J. Phys. IV France 138 (2006) 259-264

(C) EDP Sciences, Les Ulis

DOI: $10.1051 /$ jp4:2006138030

\title{
Réflectomètre à large spectre EUV pour la métrologie d'optiques
}

\author{
Ch. Hecquet ${ }^{1}$, M. Roulliay ${ }^{2}$, F. Delmotte ${ }^{1}$, M.F. Ravet-Krill ${ }^{1}$, A. Hardouin ${ }^{1}$, \\ M. Idir ${ }^{3}$ et Ph. Zeitoun ${ }^{4}$ \\ ${ }^{1}$ Laboratoire Charles Fabry de l'Institut d'Optique, CNRS, Université Paris-Sud, \\ Campus Polytechnique, RD 128, 91127 Palaiseau Cedex, France \\ ${ }^{2}$ Laboratoire d'Interaction du Rayonnement $X$ avec la Matière, UMR CNRS 8624, \\ Centre Scientifique, Bât. 350, Université Paris-Sud, 91405 Orsay, France \\ ${ }^{3}$ Synchrotron SOLEIL, L'Orme des Merisiers, Saint-Aubin, BP 48, \\ 91192 Gif-sur-Yvette Cedex, France \\ ${ }^{4}$ Laboratoire d'Optique Appliquée, ENSTA, École Polytechnique, Chemin de la Hunière, \\ 91761 Palaiseau Cedex, France
}

\begin{abstract}
Résumé. Le Laboratoire Charles Fabry conçoit de nombreuses optiques dont certaines pour les applications dans le spectre EUV. Pour les besoins de caractérisation, il est nécessaire de posséder une métrologie à la longueur d'onde d'utilisation proche des moyens de fabrication. Ceci permet d'étudier les composants dès leur conception et de caractériser les optiques. Nous présentons ici les performances d'un réflectomètre automatisé EUV large spectre. Il a été développé dans le cadre de la centrale CEMOX ${ }^{1}$, initiée par le pôle $\mathrm{PRaXO}^{2}$.
\end{abstract}

\section{INTRODUCTION}

Dans la gamme de longueurs d'onde Extrême UltraViolet (EUV = 4 à $60 \mathrm{~nm}$ ), les indices de réfraction des matériaux sont proches de celui du vide et les absorptions sont élevées ce qui constitue deux difficultés majeures pour la réalisation d'optiques. Les optiques réflectives, basées sur le principe des miroirs interférentiels multicouches, permettent d'obtenir des réflectivités importantes de l'incidence faible à la quasi-normale. L'intensité, la position et la largeur des pics de réflectivité sont les facteurs clefs à optimiser pour les applications, en particulier pour la lithographie EUV, l'analyse X et l'imagerie, dans le domaine de l'astrophysique et des diagnostics de plasma. Pour cela, il est nécessaire de posséder une métrologie à la longueur d'onde d'utilisation proche des moyens de fabrication pour étudier les multicouches dès leur conception et caractériser les optiques, avant même qu'elles soient implantées dans leur dispositif final. Nous présentons ici les caractéristiques et les performances d'un réflectomètre à large spectre fonctionnant dans le domaine EUV. Celui-ci a été développé dans le cadre de la centrale CEMOX, initiée par le pôle PRaXO. L'enceinte expérimentale offre la possibilité d'effectuer les mesures avec le goniomètre automatisé en mode de balayage en longueur d'onde et/ou en $\theta-2 \theta$ sur un large spectre d'analyse et dans un environnement propre. Il constitue un moyen de métrologie de proximité essentiel à la réalisation d'optiques EUV.

\footnotetext{
${ }^{*}$ Centrale d'élaboration de métrologie d'optiques X.

**Pôle d'optiques des Rayons-X d'Orsay.
} 


\section{LA SOURCE ET LA MISE EN FORME DU FAISCEAU}

Le système initialement implanté au laboratoire reprend le principe du réflectomètre développé au $\mathrm{CXRO}^{3}$ [1] pour les besoins de la lithographie à la longueur d'onde de $13,5 \mathrm{~nm}(92 \mathrm{eV})$ et est opérationnel entre 12-20 nm. Nous avons cherché à élargir le spectre d'utilisation.

La source plasma émet un large spectre dans la gamme de longueur d'onde comprise entre $8 \mathrm{~nm}$ et $60 \mathrm{~nm}$. Le rayonnement émis est ensuite mis en forme spatialement et spectralement pour obtenir un faisceau focalisé au centre de l'enceinte expérimentale.

\subsection{Source}

La source plasma EUV est générée à partir d'une cible métallique, qui est sous un vide de $10^{-5} \mathrm{mBar}$, sur laquelle est focalisée une impulsion laser de forte fluence. Le choix du métal de la cible détermine le spectre d'émission, et la fluence $\left(\mathrm{J} / \mathrm{mm}^{2}\right)$ détermine le nombre de photons émis par impulsion.

La source laser impulsionnelle retenue est un laser $\mathrm{Nd}$ :YAG doublé en longueur d'onde $(\lambda=532 \mathrm{~nm})$ de chez Quantel. Elle est utilisée à une fréquence de répétition comprise entre $1 \mathrm{~Hz}$ et $10 \mathrm{~Hz}$. L'énergie crête maximum pour chaque impulsion est de $400 \mathrm{~mJ}$ pour une largeur d'impulsion de $5 \mathrm{~ns}$. La pulvérisation du matériau peut être source de pollution à cause des débris de la source plasma et modifie la surface de la cible donc le plasma généré. Afin de limiter ces effets, la cible est régénérée en continue : il s'agit d'un ruban dont la tension et la vitesse de défilement sont asservies. En pratique, chaque impulsion laser interagit avec une partie vierge du ruban et génère une émission EUV dans

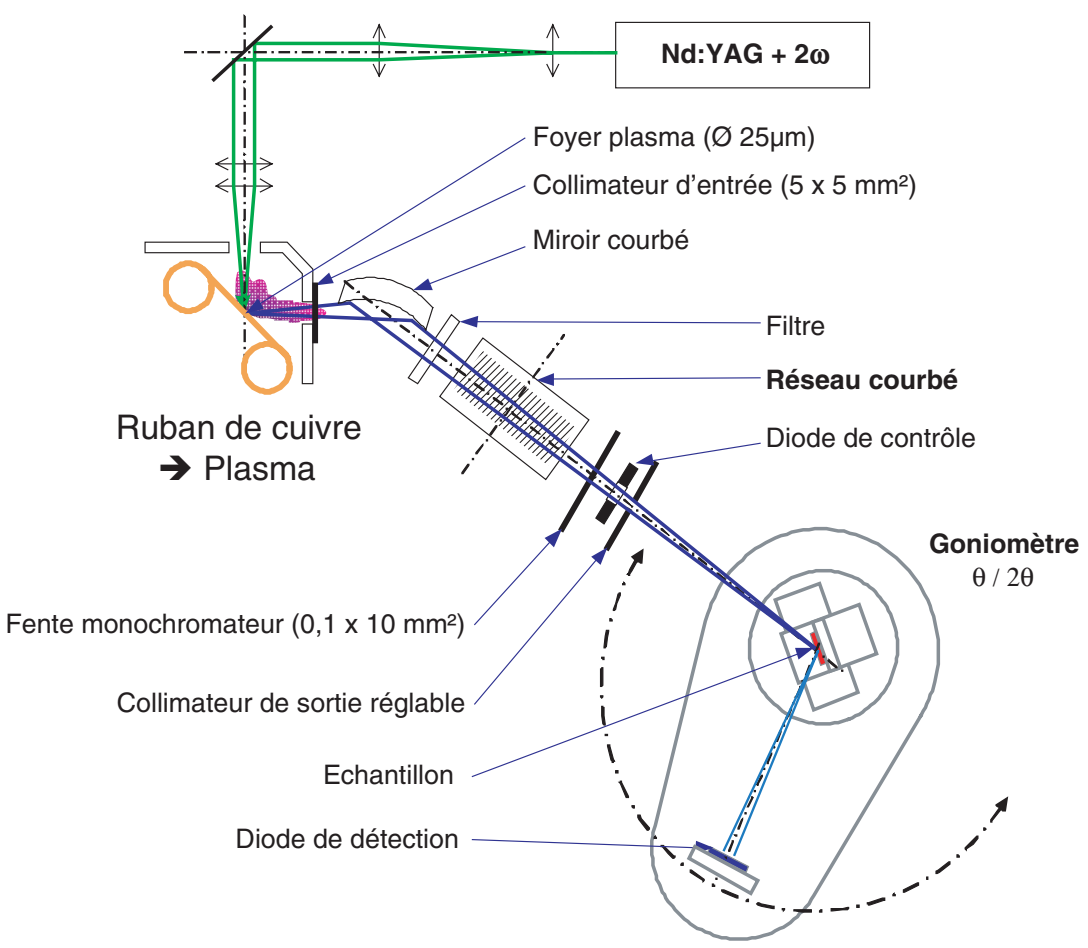

Figure 1. Schéma général du réflectomètre vu du dessus.

*** Center for X-Ray Optics de Berkeley, USA. 
$2 \pi$ stéradian, non polarisée, et les débris du matériau sont expulsés principalement derrière le ruban, limitant ainsi la pollution du premier miroir courbé.

Plusieurs métaux permettent d'obtenir des spectres d'émission autour de $13 \mathrm{~nm}: \mathrm{Al}, \mathrm{W}, \mathrm{Cu}$, Au...L'objectif étant l'obtention d'un large spectre EUV, le cuivre a été retenu. D'un point de vue expérimental, les photons émis sont détectés à partir de $8 \mathrm{~nm}$ jusqu'à $60 \mathrm{~nm}$ (limite mécanique du monochromateur).

\subsection{Mise en forme}

Le balayage en longueur d'onde est réalisé grâce à un monochromateur motorisé. Deux réseaux peuvent être utilisés : 800 traits $/ \mathrm{mm}$ (jusqu'à $\lambda=22 \mathrm{~nm}$ ) et 300 traits $/ \mathrm{mm}$ (jusqu'à $\lambda=60 \mathrm{~nm}$ ). Pour améliorer la diffraction, les réseaux sont courbés pour focaliser sur la fente de sortie et ils sont blazés pour assurer une bonne dispersion à l'ordre -1 . Le pouvoir de résolution du monochromateur dépend de l'étendue géométrique de la source et de son image. La source est le plasma lui-même dont la taille est proportionnelle à celle du faisceau laser sur la cible. Son diamètre est estimé à $25 \mu \mathrm{m}$. La fente de sortie, de $100 \mu \mathrm{m}$ de hauteur, permet la sélection spectrale. Le pouvoir de résolution calculé de ce monochromateur est représenté sur la figure 2.
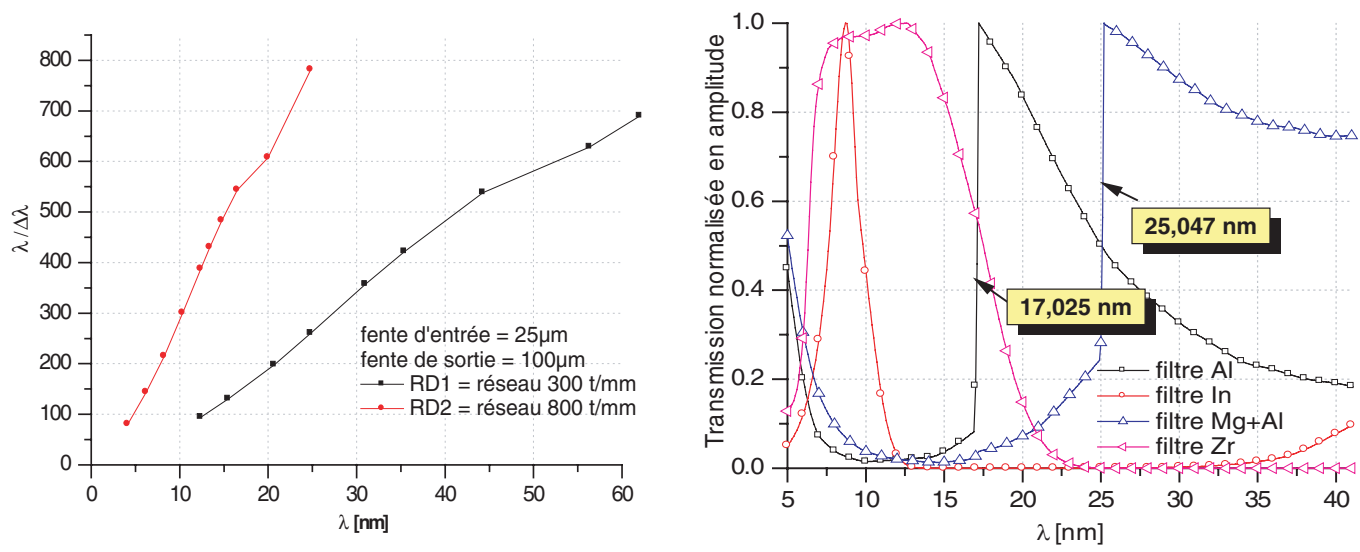

Figure 2. Pouvoir de résolution calculé du monochromateur du réflectomètre et spectres de transmission normalisés en amplitude calculés sous IMD [3] pour les différents filtres utilisés avec les seuils d'absorption de référence [4].

Le système, source - monochromateur - diode, permet de détecter des photons à partir de $8 \mathrm{~nm}$. Les longueurs d'onde supérieures présentent une combinaison de la diffraction de l'ordre -1 et des ordres supérieurs. Afin de supprimer les ordres supérieurs du réseau et la composante visible du spectre, le réseau est précédé d'un filtre métallique : $\mathrm{Al}, \mathrm{Mg}$, In ou $\mathrm{Zr}$, selon les gammes de longueur d'onde. La focalisation est réalisée en deux temps : un miroir courbe en entrée permet la focalisation sagittale, et la focalisation tangentielle est réalisée par le réseau de sortie. La section du faisceau EUV au centre de l'enceinte expérimentale est actuellement de $1 \times 10 \mathrm{~mm}^{2}$ et l'ouverture du système est de $8 \times 16 \mathrm{mrad}^{2}$.

Les performances de la source et de sa mise en forme sont reprises dans le tableau suivant.

\begin{tabular}{|l|l|}
\hline Laser & Nd:YAG doublé \\
\hline$\lambda_{\text {excitation }}$ & $532 \mathrm{~nm}(\mathrm{TON}=8 \mathrm{~ns})$ \\
\hline $\mathrm{f}_{\text {impulsion }}$ & $1 \mathrm{~Hz} \mathrm{ou} 10 \mathrm{~Hz}$ \\
\hline $\mathrm{E}_{\mathrm{Max}}$ & $400 \mathrm{~mJ}$ \\
\hline Cible & Cuivre \\
\hline $\mathrm{T}_{\mathrm{ON}} @ \mathrm{EUV}$ (après électronique de détection) & $30 \mu \mathrm{s}$ \\
\hline
\end{tabular}




\subsection{Enceinte expérimentale}

L'enceinte expérimentale est équipée d'un goniomètre. Celui-ci permet de positionner l'échantillon et le détecteur pour effectuer un balayage en $\theta-2 \theta$ avec un angle de rasance $\theta$, défini par rapport à la surface de l'échantillon, compris entre $0^{\circ}$ et $82^{\circ}$. L'ensemble fonctionne sous un vide de l'ordre de $10^{-5} \mathrm{mBar}$. Les mouvements automatisés permettent la mesure d'optiques courbes. L'échantillon est ajusté avec une précision par rapport à l'axe du faisceau grâce à 3 mouvements $\mathrm{X}$, Y et $\mathrm{Z}$ commandés par les moteurs pas à pas, avec une course de $\pm 25 \mathrm{~mm}$ respectivement. La déviation du faisceau réfléchi due à l'inclinaison de l'échantillon peut être corrigée en déplaçant la photodiode suivant l'axe $\mathrm{Zd}$ dont la course est de $\pm 15 \mathrm{~mm}$.

\section{RÉSULTATS EXPÉRIMENTAUX}

Des miroirs multicouches réalisés à l'Institut d'Optique ont été mesurés sur le réflectomètre EUV CEMOX. Différents types de substrat sont revêtus de multicouches destinées à sélectionner différentes gammes de longueur d'onde. Les paramètres recherchés sont en général la valeur de la réflectivité, à une longueur d'onde donnée, la position en longueur d'onde et la largeur à mi-hauteur. Le calage en longueur d'onde et le calcul de la réflectivité sont considérés comme deux problèmes indépendants.

Les résultats présentés sur la figure 5 montrent la réflectivité de miroirs réalisés pour l'imagerie solaire. A chaque longueur d'onde correspond une raie d'émission d'un matériau ionisé du plasma solaire, ici le FeXXIII à 13,3 nm, FeIX/X à 17,5 nm et l'HeII à 30,4 nm. Pour chaque spectre de réflectivité correspond un réseau $(\mathrm{R} 1=300 \mathrm{t} / \mathrm{mm}$ ou $\mathrm{R} 2=800 \mathrm{t} / \mathrm{mm})$ et un filtre.

La figure 3.a représente la réflectivité d'une multicouche Mo/Si optimisée à 13,3 nm mesurée sous un angle de rasance de $80^{\circ}$ avec le réseau R1 et un filtre en $\mathrm{Zr}$. La présence des pics de Kiessig montrent la bonne dynamique de mesure de l'instrument.

La figure 3.b représente la réflectivité d'une multicouche $\mathrm{Mo} / \mathrm{Si}$ optimisée à 17,5 nm mesurée à un angle de rasance de $82^{\circ}$ sur CEMOX avec le réseau $\mathrm{R} 1$ et un filtre en $\mathrm{Zr}$ ou un filtre en Al. L'amplitude avec le filtre en $\mathrm{Al}$ est proche de celle mesurée par ailleurs sur la station de métrologie du PTB [5] alors que celle avec le filtre en $\mathrm{Zr}$ est presque deux fois plus faible. La différence d'amplitude est principalement due à la contribution des ordres de diffraction supérieurs du réseau non filtrés par le filtre en Zr. On observe l'effet du bruit de mesure là où l'absorption du filtre en $\mathrm{Al}(\lambda<17,03 \mathrm{~nm})$ est maximum contrairement au filtre en $\mathrm{Zr}$.
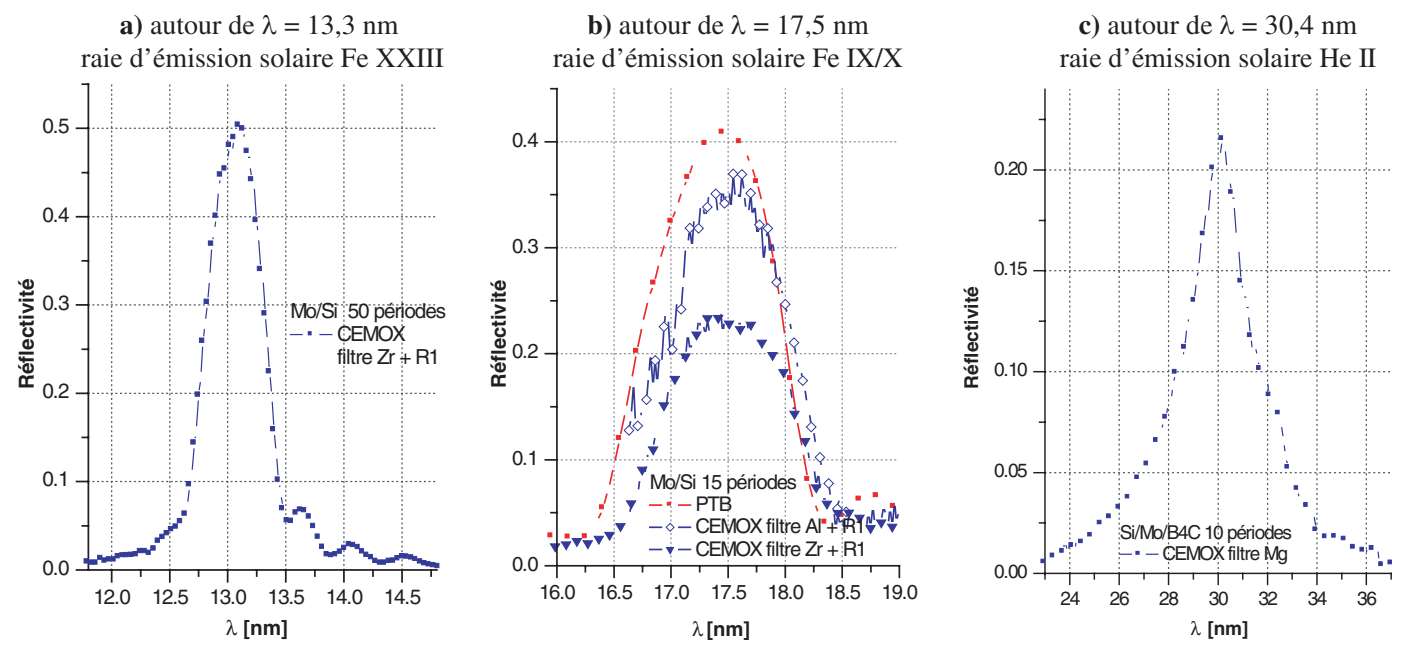

Figure 3. Réflectivité de trois structures multicouches différentes sur le réflectomètre CEMOX. 
La figure 3.c représente la réflectivité d'une multicouche $\mathrm{Si} / \mathrm{Mo} / \mathrm{B} 4 \mathrm{C}$ optimisée à $30,4 \mathrm{~nm}$ pour un angle d'incidence de $80^{\circ}$ avec le réseau R2 et un filtre en $\mathrm{Mg}$. On observe une forme proche de la théorie dont l'amplitude est néanmoins presque deux fois plus faible. Là encore, la principale cause est la pollution par des ordres de diffraction supérieurs du réseau mais aussi la polarisation qui est plus sensible avec cette structure dont nous discuterons dans la partie suivante.

\section{DISCUSSION}

\subsection{L'amplitude de la réflectivité}

Cet outil de caractérisation de laboratoire en développement permet des mesures de réflectivité sur des miroirs multicouches. La valeur de la réflectivité est obtenue dans la même gamme spectrale en calculant le rapport entre l'intensité réfléchie par la multicouche et l'intensité du faisceau direct mesuré sur le détecteur.

$$
R(\lambda)=\frac{I_{r \text { flchie }}(\lambda)}{I_{\text {transmise }}(\lambda)}
$$

La principale difficulté dans la mesure de la réflectivité, réside dans la prise en compte des effets des ordres supérieurs de diffraction. Le faisceau transmis contient les ordres supérieurs de diffraction du réseau $(<-1)$ non filtrés par le filtre métallique.

Le calcul de la réflectivité est faussé partiellement par les ordres supérieurs d'amplitude $\alpha(\mathrm{n} \lambda)$. Ils sont mesurés par le détecteur dans le faisceau transmis directement mais ne sont pas (ou peu) réfléchis par la multicouche. Ceci à pour effet de minimiser la réflectivité obtenue expérimentalement à une longueur d'onde donnée. Les filtres utilisés peuvent être considérés comme des filtres passe-bandes en longueur d'onde et donc limiter la contribution des ordres supérieurs en les filtrant. Leur pouvoir filtrant est limité par la qualité et le vieillissement du filtre qui peut être troué et donc laisser passer une partie des ordres supérieurs par des microtrous.

La réflectivité des multicouches dépend de l'angle d'incidence et de la polarisation. En polarisation s (perpendiculaire au plan d'incidence), la variation de la réflectivité est peu sensible à l'angle d'incidence alors qu'en polarisation $\mathrm{p}$, la réflectivité diminue rapidement en fonction de l'angle et de la polarisation. La réflectivité en polarisation p est quasi-nulle à l'angle de Brewster. Dans la comparaison des résultats avec les mesures effectuées avec le rayonnement synchrotron, il est nécessaire de prendre en compte la polarisation du faisceau synchrotron : majoritairement s. La source du réflectomètre est non polarisée. Ceci constitue un second paramètre de diminution de la valeur de réflectivité mesurée sur le réflectomètre, en particulier pour des angles d'incidence éloignés de la normale ou de la rasance. Dès $85^{\circ}$, par rapport au plan de l'échantillon, la simulation montre une diminution de $0.3 \%$ (en relatif) de la réflectivité par rapport à la polarisation s pour une structure $\mathrm{Mo} / \mathrm{Si}$ optimisée à $13,5 \mathrm{~nm}$. Pour le même angle, $85^{\circ}$, la réflectivité diminue de $1,9 \%$ (en relatif) pour une structure $\mathrm{Si} / \mathrm{Mo} / \mathrm{B} 4 \mathrm{C}$ optimisée à $30,4 \mathrm{~nm}$.

Pour assurer une mesure précise, il est nécessaire d'avoir une source stable. Il est préférable de mesurer simultanément la fluence incidente sur l'échantillon et celle réfléchie à même temps. La difficulté de mesure du faisceau incident est là, il n'existe pas de lame séparatrice large bande pour les EUV et le vignettage partiel du faisceau est délicat à mettre en œuvre. Actuellement, les mesures du faisceau réfléchi et du faisceau transmis sont faites à la suite. La stabilité de la source est calculée par l'écart type de plusieurs mesures. Le résultat est une répétabilité absolue de 1,5\% rms.

\subsection{La détermination de la longueur d'onde $\lambda$}

Elle est référencée par rapport aux seuils d'absorption des matériaux des filtres $\mathrm{Al}$ ou $\mathrm{Mg}$ (cf. Figure 2) et l'ordre zéro du réseau de diffraction. Le calcul du point d'inflexion de l'absorption de ces matériaux donne une position en longueur d'onde. Les seuils d'absorptions de référence utilisés ont été fixés à 
partir de la référence [4]. Le silicium a un seuil d'absorption à 12,423 nm qui est intéressant. Celui-ci ne peut être utilisé actuellement en tant que référence spectrale ou filtre spectral EUV car il transmet la composante visible du laser qui est détecté par la photodiode.

La précision du calage en longueur d'onde dépend de la reproductibilité du balayage, de la résolution du monochromateur près du seuil d'absorption et de ses ordres de diffraction supérieurs. La reproductibilité mécanique du balayage en longueur d'onde est d'environ $0,1 \mathrm{~nm}$.

Le réseau de 800 traits/mm (R1) est calibré en fonction du seuil d'absorption de l'aluminium à $17,025 \mathrm{~nm}$. La résolution calculée du réseau étant de $0,03 \mathrm{~nm}$ la précision sur la valeur du seuil sera de $\pm 0,06 \mathrm{~nm}$. D'où une précision de calage en $\lambda$ de $\pm 0,13 \mathrm{~nm}$.

Le réseau de 300 traits/mm (R2) est calibré en fonction du seuil d'absorption de l'aluminium à $17,025 \mathrm{~nm}$ ainsi que celui du magnésium à $25,047 \mathrm{~nm}$. La résolution calculée du réseau pour l'Al à la valeur du seuil est de $0,11 \mathrm{~nm}$ et respectivement de $0,096 \mathrm{~nm}$ pour le $\mathrm{Mg}$. La résolution de la valeur du seuil peut être améliorée par la mesure de la position des ordres de diffraction supérieurs du réseau soit $34,05 \mathrm{~nm}$ pour $\mathrm{Al}$ qui est résolu à $0,083 \mathrm{~nm}$ et $50,94 \mathrm{~nm}$ pour le $\mathrm{Mg}$ qui est résolu à $0,088 \mathrm{~nm}$. La précision obtenue en mesurant la valeur du seuil à l'ordre -2 de l'Al sera de $\pm 0,083 \mathrm{~nm}$. D'où une précision de calage en $\lambda$ de $\pm 0,18 \mathrm{~nm}$.

\section{CONCLUSION}

Ce réflectomètre de laboratoire dispose d'une source EUV peu polluante et stable. Il permet actuellement de vérifier le centrage en longueur d'onde de revêtements multicouches sur des miroirs et sa bonne répétabilité permet d'ajuster la position en longueur d'onde lors de la réalisation d'optiques destinées à la lithographie EUV et à l'imagerie spatiale. On devrait ainsi pouvoir observer l'évolution des optiques mesurées. L'automatisation de la source et du monochromateur permet des balayages en fonction de la longueur d'onde. Le goniomètre, lui aussi automatisé, permet de réaliser actuellement des mesures de réflectivité en $\theta-2 \theta$.

Les axes de développement actuellement envisagés sur cet appareil sont l'extension du spectre vers les longueurs d'onde plus élevée $(>40 \mathrm{~nm})$, une optimisation de la dimension du faisceau sur l'échantillon visant environ $1 \mathrm{~mm}^{2}$ et l'amélioration de la précision de la métrologie de la réflectivité par le contrôle du flux coup par coup.

\section{Remerciements}

Nous tenons à remercier Jim Underwood pour l'aide apportée, le CNRS, CNES, LNB, CEA, le conseil régional Ile de France, le Conseil Général de l'Essonne et le ministère de la recherche pour leur soutien financier au développement du réflectomètre CEMOX.

\section{Références}

[1] E. M. Guillikson, J. H. Underwood, P. C. Batson \& V. Nikitin ; A Soft X-Ray/EUV Reflectometer Based on a Laser Produced Plasma Source, J. of X-Ray Science and technology 3 (1992) 289-299

[2] L. van Loyen, T. Böttger, S. Schädlich, S. Braun, T. Foltyn, A. Leson, F. Scholze, S. Müllender; Laboratory LPP EUV reflectometer working with non-polarized radiation, Appl. Surface Science 252 (2005) 57-60

[3] D. L. Windt, Comput. Phy. 12(1998) 360

[4] E. M. Gullikson, P. Denham, S. Mrowka, and J. H. Underwood, Absolute photoabsorption measurements of $\mathrm{Mg}, \mathrm{Al}$, and $\mathrm{Si}$ in the soft-X-ray region below the L2,3 edges, Phy. Rev. B 49 (1994) no23 p16283-16288

[5] F. Scholze, J Tümmler and G Ulm, High-accuracy radiometry in the EUV range at the PTB soft X-ray beamline, Metrologia 40 (2003) S224-S228 